\title{
Tailored emission color synthesis using microfacet quantum wells consisting of nitride semiconductors without phosphors
}

\section{$\operatorname{AUTHOR}(\mathrm{S})$ :}

Funato, M; Kotani, T; Kondou, T; Kawakami, Y; Narukawa, Y; Mukai, T

\section{CITATION:}

Funato, M ... [et al]. Tailored emission color synthesis using microfacet quantum wells consisting of nitride semiconductors without phosphors. APPLIED PHYSICS LETTERS 2006, 88(26): 261920.

\section{ISSUE DATE:}

2006-06-26

URL:

http://hdl.handle.net/2433/39681

\section{RIGHT:}

Copyright 2006 American Institute of Physics. This article may be downloaded for personal use only. Any other use requires prior permission of the author and the American Institute of Physics. 


\title{
Tailored emission color synthesis using microfacet quantum wells consisting of nitride semiconductors without phosphors
}

\author{
M. Funato, ${ }^{\text {a) }}$ T. Kotani, T. Kondou, and Y. Kawakami \\ Department of Electronic Science and Engineering, Kyoto University, Kyoto 615-8510, Japan \\ Y. Narukawa and T. Mukai \\ Nitride Semiconductor Research Laboratory, Nichia Corporation, Tokushima 774-8601, Japan
}

(Received 29 March 2006; accepted 11 May 2006; published online 30 June 2006)

\begin{abstract}
A color synthesis based on InGaN/GaN quantum wells (QWs) grown on $\mathrm{GaN}$ microfacets formed by regrowth on $\mathrm{SiO}_{2}$ mask stripes is demonstrated. The microfacet structure is composed of (0001), $\{11 \overline{2} 2\}$, and $\{11 \overline{2} 0\}$ planes, and the InGaN well thickness and composition are spatially inhomogeneous due to the diffusion of the adatoms among the facets. These properties allow microfacet QWs, which, for example, emit yellow from the (0001) facet and blue from the $\{11 \overline{2} 2\}$ facet, to be fabricated, of which the luminescence appears white due to the additive color mixing. Using a mask pattern that consists of regions with and without stripes, the emissions from the microfacet QWs and from planar QWs are synthesized to produce the desired apparent output colors. (c) 2006 American Institute of Physics. [DOI: 10.1063/1.2217259]
\end{abstract}

Semiconductor optical devices emitting arbitrary colors including pastel and white impact various fields such as nanobiotechnology and solid-state lighting (SSL). ${ }^{1}$ In fact, recent developments in blue light emitting diodes (LEDs) based on $\mathrm{InGaN} / \mathrm{GaN}$ quantum wells ${ }^{2}$ (QWs) have paved the way towards white LEDs, which have attained great success in SSL. However, since semiconductor emitters inherently provide nearly monochromatic output light, present white LEDs consist of a blue LED pumping a yellow phosphor. Major drawbacks to using phosphors are that the absorption energy of a phosphor must agree with the emission energy of the LED, which can limit the material and spectral design, and that the energy loss due to the down conversion from blue to yellow is unavoidable.

Recently, we demonstrated that $\mathrm{InGaN} / \mathrm{GaN}$ QWs grown on three-dimensional $\mathrm{GaN}$ microstructures, which can be formed by a regrowth technique and consist of several microfacets with different crystalline orientations, exhibit a facet-dependent In composition and InGaN thickness. ${ }^{3,4}$ Consequently, each facet QW produces a different emission color. It is noteworthy that changing the In composition can tune the band gap energy of InGaN from $0.7 \mathrm{eV}(\mathrm{InN})$ (Ref. 5) to $3.5 \mathrm{eV}(\mathrm{GaN})$, which covers the full-visible region. Therefore, if the structural properties are controlled, then microfacet InGaN/GaN QWs can be single-chip multiwavelengths light emitters, which can control the color. Here, we demonstrate that emission light from microfacet InGaN/GaN QWs can be synthesized to produce pastel and white colors by changing the growth conditions and the mask patterns for the regrowth.

The samples were grown on sapphire (0001) substrates using low-pressure (300 Torr) metal-organic vapor phase epitaxy. Initially $\sim 5 \mu \mathrm{m} \mathrm{GaN}$ layers were grown. Then $\mathrm{SiO}_{2}$ mask stripes were formed along the $\langle 1 \overline{1} 00\rangle$ direction by a conventional photolithography. The period and opening of the stripes were 20 and $5 \mu \mathrm{m}$ wide, respectively. The subsequent regrowth of $\mathrm{GaN}$ created microstructures composed of

${ }^{a)}$ Electronic mail: funato@kuee.kyoto-u.ac.jp
(0001), $\{11 \overline{2} 2\}$, and $\{11 \overline{2} 0\}$ facets along the $\langle 1 \overline{1} 00\rangle$ direction, similar to Refs. 6 and 7. Finally, InGaN/GaN five-period QWs were fabricated on these microfacets. For the QW growth, the molar flow ratio between group III sources and ammonia (V/III ratio) was 5500, and the temperature was around $750-800{ }^{\circ} \mathrm{C}$.

The local InGaN thickness and In composition on the microfacets were evaluated prior to fabricating the QWs. Preliminary experiments for planar (0001) QWs indicate that the growth rates of $\mathrm{InGaN}$ in the QWs and the thick InGaN are the same, although the In compositions are different. Therefore, InGaN thick films and $\mathrm{InGaN} / \mathrm{GaN} 200$ period superlattices were grown on $\mathrm{GaN}$ microfacets for thickness and composition analyses, respectively. The measurements were performed in the $\langle 1 \overline{1} 00\rangle$ direction by scanning electron microscopy (SEM) and energy dispersive x-ray spectroscopy (EDS) equipped to the SEM. Figure 1 shows the results. The cross sectional SEM image shown in the inset confirms that the (0001), $\{11 \overline{2} 2\}$, and $\{11 \overline{2} 0\}$ microfacets are formed on the top, slopes, and sidewalls, respectively. It is found that the growth rate increases in the order of $(0001)>\{11 \overline{2} 0\}$

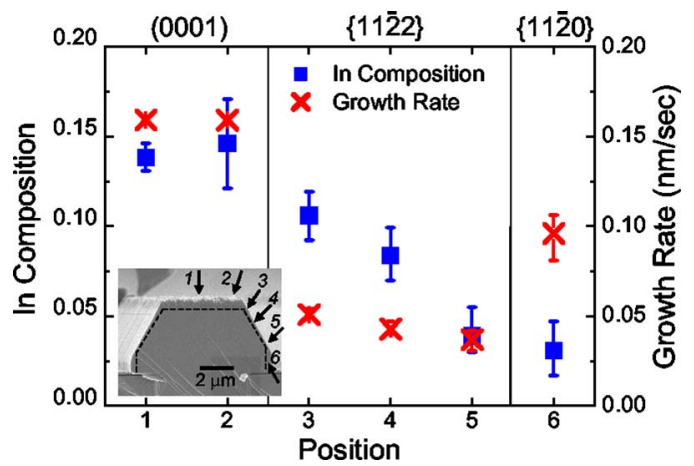

FIG. 1. (Color online) Local InGaN growth rate and In composition. The inset is a cross sectional SEM image viewed along the $\langle 1 \overline{1} 00\rangle$ direction. The numbers in the image designate the positions where the growth rate and In composition are estimated. 
$>\{11 \overline{2} 2\}$ and that the growth rate on the $\{11 \overline{2} 2\}$ facet is faster for the (0001) side. On the other hand, the In composition increases from the $\{11 \overline{2} 0\}$ facet to $\{11 \overline{2} 2\}$ facet, reaches a maximum at the border between the $\{11 \overline{2} 2\}$ and (0001) facets (position 2), and decreases on the (0001) facet as it approaches the center (from position 2 to 1). Planar (0001) QWs grown under the same growth conditions have a growth rate of $0.137 \mathrm{~nm} / \mathrm{s}$ and an In composition of 0.13 , which are both lower than on the (0001) facet and higher than on the other facets. ${ }^{8}$

These experimental findings suggest that the diffusivity of surface adatoms during growth is in the order of $\{11 \overline{2} 2\}>\{11 \overline{2} 0\}>(0001)$, which differs for Ga and In. Since $\{11 \overline{2} 2\}$ is a nitrogen terminated plane, the high diffusivity and the consequent slow growth rate on the $\{11 \overline{2} 2\}$ facet are probably due to the lack of nitrogen precursors on the facet surface caused by the diffusion of Ga and In adatoms from the mask regions. On the other hand, the various growth rates on the $\{11 \overline{2} 2\}$ facet indicate that group III adatoms chiefly migrate toward the (0001) facet, while the variation of the In composition on the same facet indicates that In possesses a higher diffusivity than Ga due to its higher vapor pressure. The highest In composition is on the border between (0001) and $\{11 \overline{2} 2\}$, which can be understood by considering that In atoms migrating from $\{11 \overline{2} 2\}$ to (0001) remain on the border due to the lower diffusivity on the (0001) facet. It should be noted that the growth properties discussed here are true for other samples with different mask geometries, although the dimensions of the microstructures, the InGaN thickness, and the In composition change. Thus, mask geometry can also control QW energy levels.

In addition to the well width and composition discussed above, the polarization effects ${ }^{9-11}$ must be considered in order to design the emission color from microfacet QWs. In this study, we calculated the piezoelectric polarization with the consideration of the growth direction ${ }^{10,11}$ using the latest physical parameters. Then the quantized ground level in the presence of piezoelectric and spontaneous polarizations, which corresponds to the emission color, was calculated.

Two microfacet QWs that emitted different colors were designed. One emitted $500 \mathrm{~nm}$ from the (0001) facet and $400 \mathrm{~nm}$ from the $\{11 \overline{2} 2\}$ facet (sample A), while the other emitted $580 \mathrm{~nm}$ from the (0001) facet and $430 \mathrm{~nm}$ from the $\{11 \overline{2} 2\}$ facet (sample B). The In composition in the $\{11 \overline{2} 0\}$ facet was insufficient to emit visible light. Hence, the contribution from this facet was neglected. To confirm the emission color from each facet of the fabricated microfacet QWs, the microscopic optical properties were examined at room temperature (RT) by cathodeluminescence (CL) measurements with an acceleration voltage of $5 \mathrm{kV}$. Figure 2 shows the obtained spectra. As shown in the inset, the measurements were performed in the [0001] direction at three different positions $a, b$, and c. Position a was on the $\{11 \overline{2} 2\}$ facet, while positions $b$ and $c$ were on the (0001) facet. It can be seen that the emission wavelengths were consistent with the design. Although positions b and c were on the same facet, their emissions peaked at different wavelengths. This can be understood by considering the In distribution shown in Fig. 1. The higher In compositions at the border between Downloaded 30 May 2007 to 130.54 .110 .22 . Redistribution subject

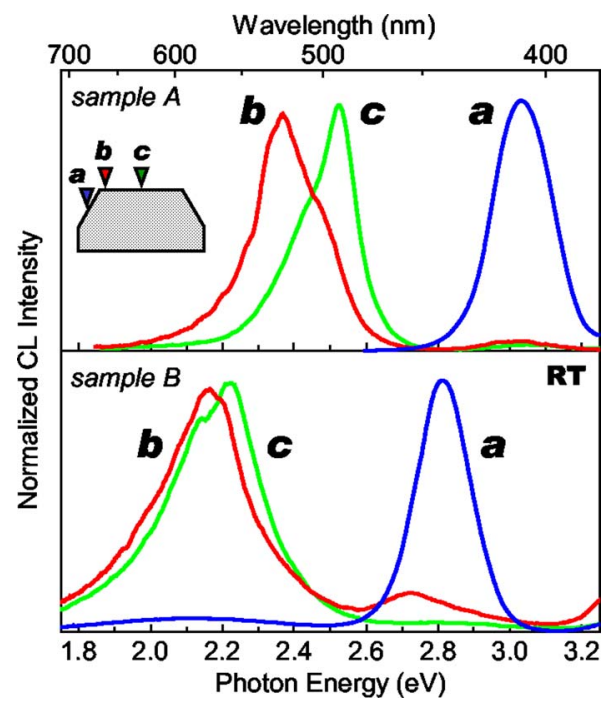

FIG. 2. (Color online) CL spectra of samples A and B acquired at RT. The inset is a schematic of the cross section of the microfacet QW and indicates the measurement positions $\mathrm{a}, \mathrm{b}$, and $\mathrm{c}$.

(0001) and $\{11 \overline{2} 2\}$ result in longer emission wavelengths for position $b$.

The macroscopic optical properties of the microfacet QWs were assessed by photoluminescence (PL) measurements at RT. Figures 3(a) and 3(b) show the PL spectra and photographs of the emitting samples. The excitation source was a $\mathrm{He}-\mathrm{Cd}$ laser with an excitation density of $10 \mathrm{~W} / \mathrm{cm}^{2}$ and an excited spot of $300 \mu \mathrm{m}$, which included many (0001) and $\{11 \overline{2} 2\}$ facets. The origins of the emissions, indicated in Figs. 3(a) and 3(b), were identified using the CL spectra (Fig. 2). A characteristic of the PL spectra was the rather broad linewidth, which was caused by the In spatial distribution within a facet. Particularly, the shoulders observed for the emission from the (0001) QWs reflected the spatial inhomogeneity revealed by the CL measurement (Fig. 2). This broad linewidth can improve the color rendering properties.

The insets in Figs. 3(a) and 3(b) show the appearances of the emissions. The two emission bands observed in the PL spectra were mixed and the apparent colors were greenish white for A and purplish white for B, indicating color syntheses of the nearly monochromatic light output from each facet QW. In terms of the Commission Internationale de I'Éclairage (CIE) chromaticity diagram, the emission of A was at $(0.216,0.453)$ while that of $\mathrm{B}$ was at $(0.318,0.262)$, which are plotted as the open circles in Fig. 3(c). Monochromatic light from conventional LEDs should be on the boundary in the CIE chromaticity diagram. In contrast, the emissions of our microfacet QWs remained far from the boundary and were classified into green for A and white for B, indicating that pastel and white colors can be realized by nitride semiconductors without phosphors.

In $\mathrm{A}$ and $\mathrm{B}$, changing the growth conditions controlled the output colors, while the mask geometry also changed the InGaN thickness and In composition, which consequently altered the emission color. Here, in a preliminary experiment, masks, which were composed of regions with and without stripes, were examined since it was predicted that the emission shown in Figs. 3(a) and 3(b) was synthesized with the emission from conventional planar QWs grown on the stripeless region. Without the mask stripes, the PL peaked at to AIP license or copyright, see http://apl.aip.org/apl/copyright.jsp 


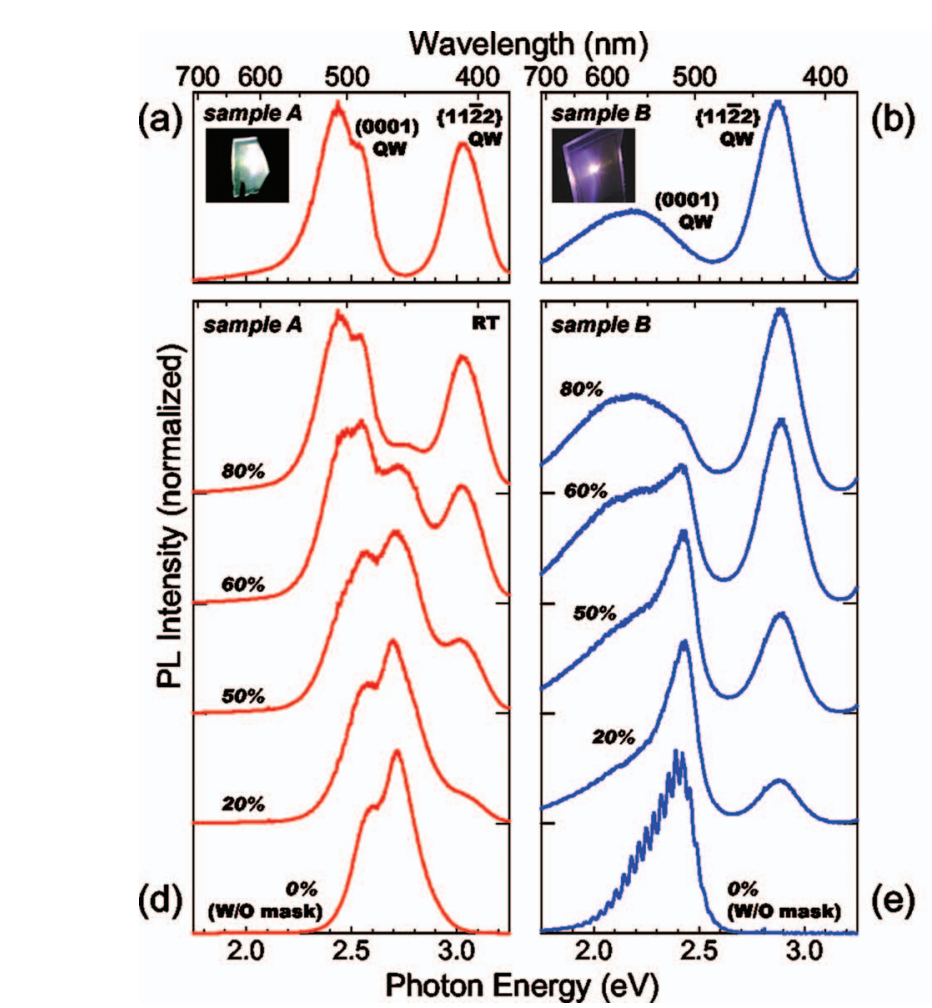

(c)

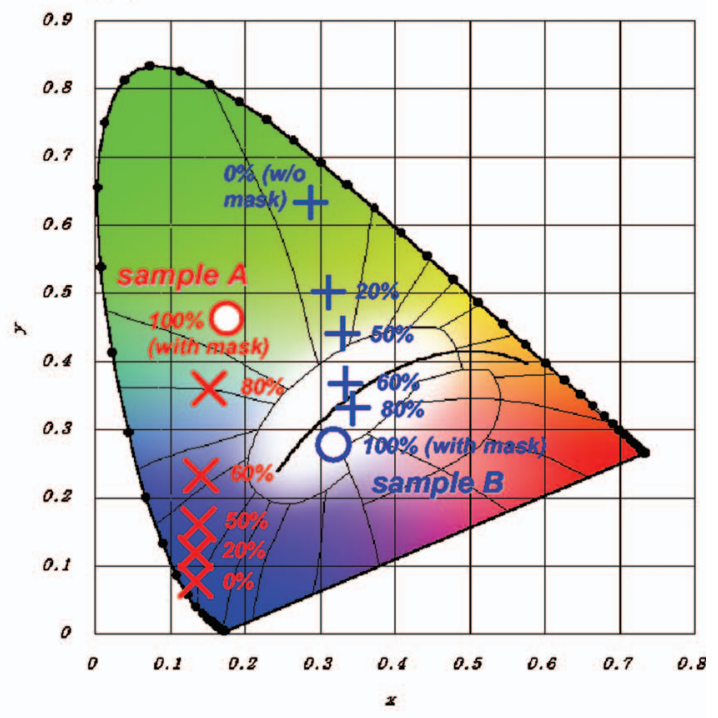

FIG. 3. (Color) PL spectra of samples A and B acquired at RT and CIE chromaticity diagram. (a) PL spectrum of sample A. The inset photograph shows sample A that emits a whitish color. (b) Those for sample B. (c) CIE chromaticity diagram, where the open circles indicate the emissions from samples A (a) and B (b), while the crosses indicate the emissions from the samples with designed mask patterns. See text for more details. (d) Variation of PL spectra due to mask geometries for sample A. (e) That for sample B.

$460 \mathrm{~nm}$ for $\mathrm{A}$ and $520 \mathrm{~nm}$ for $\mathrm{B}$, which were both between the emissions from the (0001) and $\{11 \overline{2} 2\}$ microfacet QWs, as shown at the bottom of Figs. 3(d) and 3(e). The changes in the PL spectra as a proportion of the masked region varied from $0 \%$ to $100 \%$ were measured at RT and are shown in Figs. 3(d) and 3(e), where the percentages in italics indicate the proportions. The spectral synthesis by changing the mask geometries can clearly be recognized. The crosses in Fig. 3 (c) plot the results in terms of the CIE chromaticity diagram. The output light color was easily controlled from blue to green through bluish green and greenish blue for A and from yellowish green to white for B.

We demonstrated the emission color synthesis using the microfacet InGaN/GaN QWs fabricated by the regrowth technique without phosphors. The apparent color is controlled by the growth conditions and mask geometries. It is these observations that lead us to believe that our proposed structure is promising for light emitting devices, which require sophisticated syntheses of colors. In addition, the size, which is a micron or smaller, should enable micro- or nanolight emitters, which can monolithically be integrated with other devices to create highly functional systems, to be realized.
${ }^{1}$ J. Tsao, IEEE Circuits Devices Mag. 20, 28 (2004).

${ }^{2}$ S. Nakamura, S. Pearton, and G. Fasol, Blue Laser Diode, 2nd ed. (Springer, Heidelberg, 2000).

${ }^{3}$ K. Nishizuka, M. Funato, Y. Kawakami, Sg. Fujita, Y. Narukawa, and T. Mukai, Appl. Phys. Lett. 85, 3122 (2004).

${ }^{4}$ K. Nishizuka, M. Funato, Y. Kawakami, Y. Narukawa, and T. Mukai, Appl. Phys. Lett. 87, 231901 (2005).

${ }^{5}$ J. Wu, W. Walukiewicz, K. M. Yu, J. W. Ager III, E. E. Haller, H. Lu, and W. J. Schaff, Phys. Status Solidi B 240, 412 (2003).

${ }^{6}$ H. Marchand, J. P. Ibbetson, P. T. Fini, S. Keller, S. P. DenBaars, J. S. Speck, and U. K. Mishra, J. Cryst. Growth 195, 328 (1998).

${ }^{7}$ K. Hiramatsu, K. Nishiyama, M. Onishi, H. Mizutani, M. Narukawa, A. Motogaito, H. Miyake, Y. Iechika, and T. Maeda, J. Cryst. Growth 221, 316 (2000).

${ }^{8}$ The difference of the In composition and InGaN thickness on planar (0001) QWs and facets has been discussed in T. Takeuchi, S. Lester, D. Basile, G. Girolami, R. Twist, F. Mertz, M. Wong, R. Schneider, H. Amano, and I. Akasaki, IPAP Conf. Ser. 1, 137 (2000); B. Neubert, P. Brückner, F. Habel, F. Scholz, T. Riemann, J. Christen, M. Beer, and J. Zweck, Appl. Phys. Lett. 87, 182111 (2005).

${ }^{9}$ F. Bernardini, V. Fiorentini, and D. Vanderbilt, Phys. Rev. B 56, R10024 (1997).

${ }^{10}$ T. Takeuchi, H. Amano, and I. Akasaki, Jpn. J. Appl. Phys., Part 1 39, 413 (2000).

${ }^{11}$ S.-H. Park, J. Appl. Phys. 91, 9904 (2002). 